\title{
Deletion of the Phytophthora sojae Avirulence Gene Avr1d Causes Gain of Virulence on Rps1d
}

\author{
Ren Na, ${ }^{1,2}$ Dan Yu, ${ }^{1,3}$ Dinah Qutob, ${ }^{1}$ Jun Zhao, ${ }^{2}$ and Mark Gijzen ${ }^{1}$ \\ ${ }^{1}$ Agriculture and Agri-Food Canada, 1391 Sandford Street, London, ON, N5V 4T3, Canada; ${ }^{2}$ College of Agronomy, Inner \\ Mongolia Agricultural University, Huhhot 010019, China; ${ }^{3}$ College of Plant Protection, Nanjing Agricultural University, \\ Nanjing 210095, China
}

Submitted 1 February 2013. Accepted 22 March 2013.

\begin{abstract}
Phytophthora sojae is an oomycete and a pathogen of soybean that causes root rot. During infection $P$. sojae delivers effector proteins into host cells to foster disease. However, effector-triggered immunity (ETI) results when pathogen factors are recognized by host resistance $(R)$ proteins. We have now identified the $P$. sojae Avrld gene, which encodes a predicted effector protein with the amino acid motif ArgX-Leu-Arg (RXLR). Genetic mapping of 16 different $P$. sojae isolates and of a segregating $\mathrm{F}_{2}$ population of 40 individuals shows that the predicted RXLR effector gene Avh6 precisely cosegregates with the Avrld phenotype. Transient expression assays confirm that Avrld triggers cell death specifically in Rps1d soybean plants. The Avrld gene is present in $P$. sojae strains that are avirulent on Rps 1d, whereas the gene is deleted from the genome of virulent strains. Two sequence variants of the Avrld gene encoding different protein products occur in $P$. sojae strains, but both are recognized by Rps1d and cause ETI. Liposome binding assays show that Avr1d has affinity for phosphatidylinositol 4-phosphate and that binding can be disrupted by mutation of lysine residues in the carboxy-terminal effector domain of the protein. The identification of Avrld aids pathogen diagnostics and soybean cultivar development.
\end{abstract}

Plant pathogens must overcome preformed and induced defenses in order to successfully colonize and reproduce on host plants. Induced host-plant defenses are activated by danger signals that arise from pathogen growth (Thomma et al. 2011). Effector proteins are secreted by pathogens to suppress host defenses and foster disease (Bozkurt et al. 2012). Nonetheless, pathogen effectors can become self-defeating when plant immune systems recognize their presence. This interaction can be genetically tracked in the host and pathogen. In such cases, host plant resistance $(R)$ genes and pathogen avirulence $(A v r)$ genes encode the immune and effector system components that determine the disease outcome, either resistance or susceptibility (Jones and Dangl 2006).

Nucleotide sequence data are available in the GenBank database under accession numbers KC004057 to KC004058.

Corresponding author: Mark Gijzen; E-mail: mark.gijzen@agr.gc.ca

* The $\boldsymbol{e}$-Xtra logo stands for "electronic extra" and indicates that five supplementary figures and one supplementary table are published online. Also, Figures 2, 4, and 5 appear in color online.

This article is in the public domain and not copyrightable. It may be freely reprinted with customary crediting of the source. The American Phytopathological Society, 2013.
The interaction between soybean and the root rot pathogen Phytophthora sojae follows the $R$ - and Avr-gene model (Gijzen and Qutob 2009). Soybean $R$ genes that confer strainspecific resistance to $P$. sojae are known as Rps genes. Complementary $P$. sojae Avr genes encode effector proteins that potentially can elicit rapid and robust host immune responses via a process known as effector triggered immunity (ETI). The identification of $A v r$ genes from $P$. sojae and other oomycete plant pathogens provides practical tools for crop breeding, pathogen diagnostics, and disease management (Vleeshouwers et al. 2011; Rietman et al. 2012). Known $P$. sojae Avr genes include Avrla (Qutob et al. 2009), Avrlb (Shan et al. 2004), Avr3a/5 (Dong et al. 2011a), Avr3b (Dong et al. 2011b), Avr3c (Dong et al. 2009), and $A v r 4 / 6$ (Dou et al. 2010). Each of these $A v r$ genes is predicted to encode a secreted protein containing an RXLR (Arg-any amino acid-Leu-Arg) and dEER (Asp-GluGlu-Arg) motif downstream from the signal peptide. The RXLR and associated dEER motif are proposed to function in targeting the effector for delivery to the host cell cytoplasm (Dou et al. 2008; Whisson et al. 2007). Whole genome analysis has demonstrated that RXLR effectors constitute huge and rapidly evolving gene families in Phytophthora species (Jiang et al. 2008; Win et al. 2007). For example, the P. sojae and Phytophthora infestans genomes each possess some 350 to 560 distinct genes encoding RXLR effectors (Haas et al. 2009; Tyler et al. 2006). Predicted $P$. sojae RXLR effectors that are not known to be Avr genes are named Avirulence homolog (Avh) genes (Wang et al. 2011).

Secreted RXLR effectors have the ability to enter host cells, but it is not clear how this occurs. Some investigators have suggested that binding to phospholipids such as phosphatidylinositol 3-phosphate [PtdIns(3)P] is important for host cell targeting (Kale et al. 2010), while others have discounted this hypothesis (Wawra et al. 2012). The RXLR motif was proposed to function in PtdIns(3)P binding (Kale et al. 2010), but subsequent results indicate that most of the lipid-binding affinity resides downstream in the carboxy $(\mathrm{C})$-terminal effector domain of the protein rather than the RXLR sequence (Sun et al. 2013; Wawra et al. 2012; Yaeno et al. 2011).

The purpose of the present study was to identify the $P$. sojae Avrld gene. This was accomplished by genetic mapping and functional genomic approaches. The P. sojae Avrld gene corresponds to a predicted protein with a signal peptide and an RXLR motif. Deletions of the Avrld structural gene cause gain of virulence in $P$. sojae strains that are able to defeat the soybean Rps 1d resistance gene. Expression and purification of recombinant Avr1d protein was performed to test the lipidbinding properties of the protein. Results indicate that Avr1d binds to phosphatidylinositol-4-phosphate [PtdIns(4)P] rather 
than to PtdIns(3)P. Lipid binding could be disrupted by mutation of selected lysine residues in the C-terminal effector domain but not by mutation of the RXLR motif.

\section{RESULTS}

\section{Avrld segregates as a single dominant gene} in $P$. sojae cross ACR10 $\times$ P7076.

To test for the segregation of the predicted Avrld gene, we performed a cross between $P$. sojae strains ACR10 (avirulent on Rps1d) and P7076 (virulent on Rps1d). A total of $20 \mathrm{~F}_{1}$ hybrids were tested, and each displayed avirulence towards Rps 1 d. A population of 40 individuals developed from one $\mathrm{F}_{1}$ progeny was used to determine the segregation of Avrld in the $\mathrm{F}_{2}$ generation. Virulence segregated as a qualitative trait with the ratio of avirulent/virulent $F_{2}$ cultures being 32:8. This ratio is consistent with the expected value of $3: 1$, as predicted for a single dominant Avrld gene (chi-squared probability $=0.46$ ).

\section{The predicted RXLR effector Avh6 cosegregates with Avrld.}

Known Avr genes from Phytophthora species possess common characteristics that provide a starting point for searches of unidentified Avr genes. To begin the hunt for Avrld, we made the following hypotheses. It encodes a secreted protein with RXLR motif; it is expressed in avirulent strains of the pathogen; and it is polymorphic in sequence or expression between strains that differ in virulence on plants with Rps 1d. A total of 497 different $A v h$ genes have been identified from wholegenome sequence data of $P$. sojae reference strain P6497 (Wang et al. 2011). Transcript analysis provides evidence for expression of 119 of these predicted RXLR effectors. Genomic resequencing of three additional $P$. sojae strains, P7064, P7074, and P7076, indicates that expressed Avh sequences are often polymorphic (Wang et al. 2011). Strains that differ in their virulence phenotype to particular Rps genes would be expected to display corresponding differences in the sequence or expression of the corresponding Avr gene. Thus, by analysis of Avh gene expression and sequence polymorphisms and comparison with strainspecific virulence on particular Rps genes, candidate $A v r$ effectors can be identified a priori.

Table 1. Testing of 15 candidate Avirulence homolog (Avh) genes for cosegregation with Avrld

\begin{tabular}{lcclc}
\hline & \multicolumn{3}{c}{ P. $^{*}$ sojae strain $^{\mathbf{a}}$} & \\
\cline { 2 - 4 } Candidate & P7064 $^{\mathbf{b}}$ & P7074 $^{\mathbf{c}}$ & P7076 $^{\mathbf{c}}$ & Avrld locus $^{\mathbf{d}}$ \\
\hline$A v h 6$ & SP & Absent & Absent & Linked \\
$A v h 15$ & SP & NP & SP & Unlinked \\
$A v h 20$ & NP & SP & SP & Unlinked \\
$A v h 51$ & NP & NP & SP & Unlinked \\
$A v h 67$ & NP & SP & SP & Unlinked \\
$A v h 71$ & NP & SP & SP & Unlinked \\
$A v h 161$ & NP & NP & SP & Unlinked \\
$A v h 186$ & ND & SP & SP & Unlinked \\
$A v h 225$ & NP & NP & NP & Unlinked \\
$A v h 238$ & NP & SP & SP & Unlinked \\
$A v h 288$ & ND & SP & SP & Unlinked \\
$A v h 308$ & SP & SP & SP & Unlinked \\
$A v h 320$ & SP & NP & SP & Unlinked \\
$A v h 343$ & NP & SP & NP & Unlinked \\
$A v h 362$ & NP & NP & SP & Unlinked \\
\hline
\end{tabular}

${ }^{a}$ SP, sequence polymorphisms between tested strain and reference strain P6497; Absent, sequence absent in the tested P. sojae strain; NP, no sequence polymorphisms between tested strain and reference strain P6497; ND, not determined

${ }^{\mathrm{b}}$ P. sojae strain avirulent on Rps $1 \mathrm{~d}$.

${ }^{\mathrm{c}} P$. sojae strain virulent on Rps $1 \mathrm{~d}$.

${ }^{\mathrm{d}}$ Result of linkage analysis for segregation of candidate Avh genes and Avrld phenotype using ACR $10 \times \mathrm{P} 7076 \mathrm{~F} 2$ progeny.
This rationale guided our selection of 15 predicted RXLR effectors as potential candidate genes for Avrld (Table 1). These 15 candidate genes display strain-specific polymorphisms that are consistent with a possible role in determining virulence on plants carrying Rps $1 \mathrm{~d}$; however, not all of the candidates were among the 119 RXLR effectors with evidence for expression. Molecular DNA markers based upon presence or absence of amplified sequences or upon cleaved amplified polymorphic sequences were developed for each of the 15 candidate $A v h$ genes. The DNA markers were validated as polymorphic in parental strains ACR10 and P7076 prior to testing the 40 progeny of the segregating $F_{2}$ population. Results from this analysis indicate that the predicted RXLR effector gene Avh6 cosegregates with Avrld (Table 1; Supplementary Fig. S1). The Avh6 gene lies on a contiguous sequence named Scaffold_5 in the P. sojae reference genome assembly V5.0.

It was necessary to further characterize the region around $A v h 6$ to verify that the reference genome assembly from $P$. sojae P6497 provides a useful physical map for segregation of this gene in ACR10 $\times$ P7076. Furthermore, we noted that four other predicted RXLR effector genes, Avh73, Avh272, Avh282 and $A v h 283$, occur in the vicinity of Avh6 in the reference genome. We sought to create a fine genetic map of the region to delineate the segment containing Avrld, preferably using more informative codominant markers. The Avh6 DNA marker is not optimal for mapping because it is a dominant marker that detects presence or absence of the gene and cannot distinguish heterozygous- from homozygous-dominant genotypes. Thus, an additional 28 codominant DNA markers flanking the Avh6 gene were developed. The DNA markers were designed based upon sequence polymorphisms between $P$. sojae reference strain P6497 and resequenced strain P7076 and were validated by scoring in the parental strains ACR10 and P7076. Results from this analysis are shown in Figure 1 and Supplementary Figure S2. The 28 DNA markers flanking Avh6 produced a genetic map in the ACR10 $\times$ P7076 $\mathrm{F}_{2}$ progeny that is consistent with their physical location in reference genome P6497. With the exception of $A v h 6$, which cosegregates with Avrld, all other Avh genes in the region display recombination with Avrld. Furthermore, analysis of $A v h 73, A v h 272$, and $A v h 283$ for sequence differences indicates that these predicted RXLR effector genes are not good candidates for Avrld because they do not display any polymorphisms among four $P$. sojae strains that differ in virulence against Rps1d (Wang et al. 2011). The Avh282 gene is polymorphic and is among the 119 RXLR effectors with evidence for expression (Wang et al. 2011), but this gene does not cosegregate with Avrld. Thus, the Avh6 gene remains the best candidate for Avrld because it precisely cosegregates with Avrld and it displays the presence or absence polymorphisms that are consistent with Rps1d virulence phenotypes in the reference and resequenced strains of $P$. sojae.

\section{Haplotype and transcript analysis of Avh6 matches Avrld phenotypes among $P$. sojae strains.}

Whole-genome sequence information for four different $P$. sojae strains suggested that the Avh6 gene is polymorphic in coding sequence between strains P6497 and P7064 and that it additionally displays presence or absence polymorphisms among strains (Table 1). These observations were verified by analysis of 16 different $P$. sojae strains within our own collection, as shown in Figure 2. The Avh6 gene encodes a predicted protein of 125 amino acids, including a signal peptide, RXLRdEER sequences, and a W-motif, as shown in Figure 2A. Using the Avh6 DNA marker to score for the presence or absence of this gene in the 16 strains, we found that 11 strains carry a copy of Avh6 and are avirulent against Rps $1 \mathrm{~d}$, whereas five strains are lacking an Avh6 gene and are virulent towards Rps 1d 
(Fig. 2B). Haplotype mapping of Avh6 and associated genetic markers from the 16 strains provides further evidence that Avh6 corresponds to Avrld (Supplementary Fig. S3). Among the 11 strains carrying a copy of $A v h 6$, two sequence variants of $A v h 6$ were identified that encoded slightly different protein products (Fig. 2A). The most variable region of the protein was found within the effector domain. The Avh6-1 allele was present in seven strains, while the Avh6-2 allele was found in four strains (Fig. 2B).

To test for the presence of the Avh6 transcript, reverse transcriptase polymerase chain reaction (RT-PCR) was performed, using template mRNA isolated from the $16 P$. sojae isolates within our collection. As expected, transcripts of Avh6 are present in strains avirulent towards Rps $1 \mathrm{~d}$ but not in the corresponding virulent strains (Fig. 2B).

\section{Transient expression}

in soybean confirms that Avh6 is Avrld.

Effector proteins expressed by $P$. sojae Avr genes trigger cell death in soybean plants that carry matching Rps genes. In order to test whether Avh6 can trigger cell death in an Rps 1d soybean plant, transient expression of Avh6 together with a reporter gene was performed in a cobombardment assay. Plasmid constructs of Avh6 excluding the signal peptide were bombarded into soybean leaves together with the glucoronidase $(G U S)$ gene to measure cell death. This was done for each of the Avh6 alleles, Avh6-1 and Avh6-2, in the soybean isolines Harosoy (rps1d) and Haro16 (Rps1d). Expression of each of the Avh6 alleles triggered cell death in Haro16 (Rps1d) but not in Harosoy (rps1d) leaves, as shown in Figure 3A and Supplementary Figure S4. Results from this

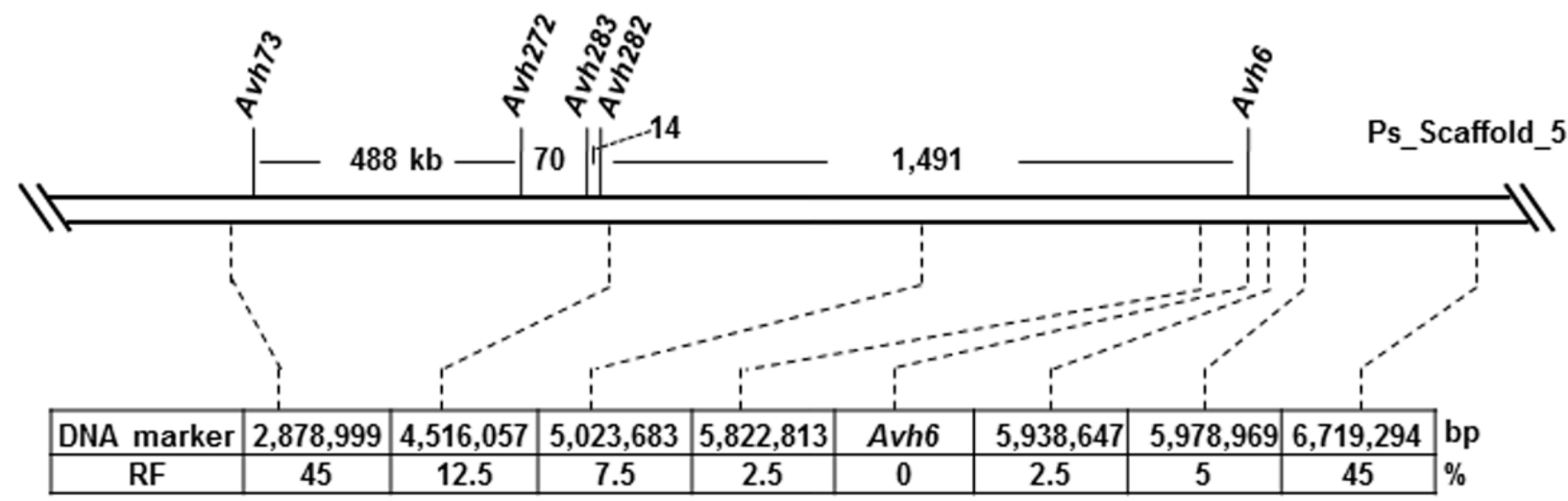

Fig. 1. Physical and genetic map of the Avh6 region. The linear map shows the position of Avirulence homolog (Avh) genes occurring on a segment of Phytophthora sojae Scaffold_5 (assembly V5.0). Scale is in kilobase pairs (kb). The table below provides the exact position of selected DNA markers in base pairs (bp) and the corresponding recombination frequencies (RF) with Avrld phenotype within the $\mathrm{F}_{2}$ population.

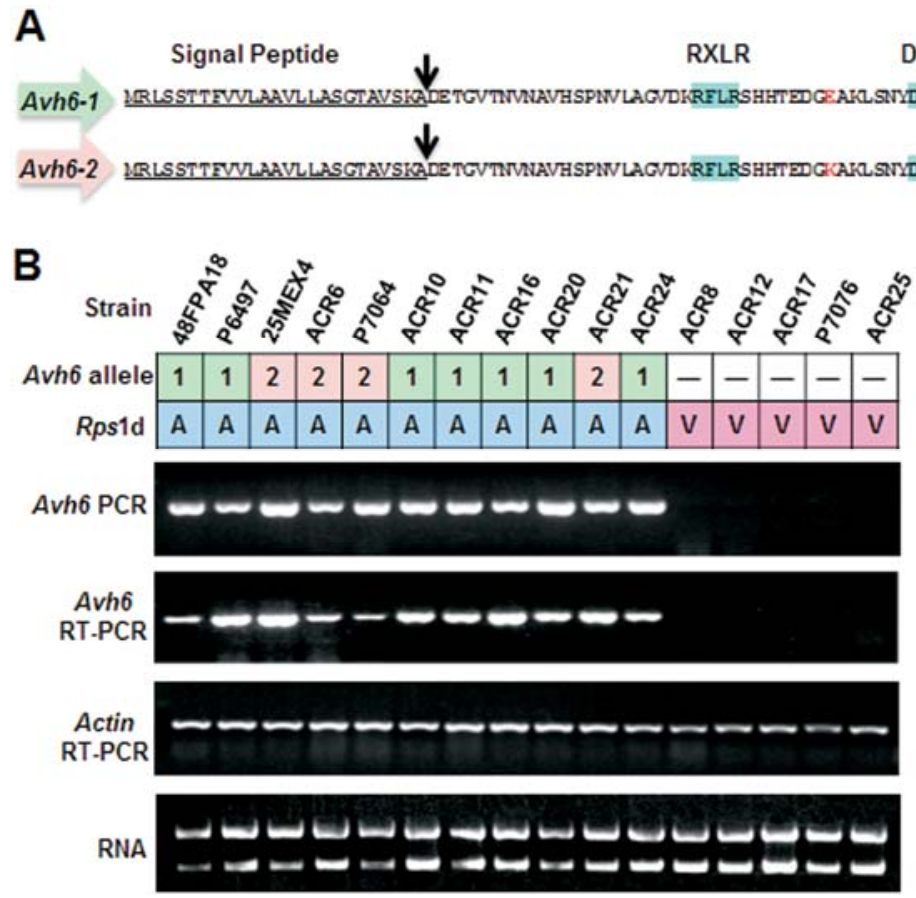

Fig. 2. The predicted RXLR effector Avh6 corresponds to Avrld. A, Primary sequence of Avh6 alleles. Two sequence variants of Avh6 were identified among Phytophthora sojae strains, as shown. The signal peptide domain is underlined. Black arrows denote the putative signal peptide cleavage site. The RXLR dEER motif is highlighted. The W-like domains are boxed with signature residues indicated below each protein. B, Expression of Avh6 in Phytophthora sojae strains cosegregates with Avrld phenotype. Virulence of P. sojae strains on Rpsld plants is shown as virulent (V) or avirulent (A). Polymerase chain reaction (PCR) analysis was performed on genomic DNA to test for the presence of the Avh6 gene in different $P$. sojae strains. Reverse transcriptase-PCR (RT-PCR) analysis was performed using cDNA from mRNA, to test for transcripts of Avh6 and a control gene (Actin). Samples of total RNA isolated from each strain, showing the small and large subunit of rRNA, are also shown (RNA). 
functional assay indicate that there is a specific interaction between Avh6 and Rps1d, and we conclude that Avh6 corresponds to Avrld.

To determine the influence of the RXLR motif on host targeting, as assessed by the cobombardment assay, we tested Avr1d constructs with the RFLR (Arg-Phe-Leu-Arg) sequence mutated to AAAA (Ala-Ala-Ala-Ala). This was performed for each of the two Avr1d open reading frames, with the signal peptides intact. Results shown in Figure 3B and Supplementary Figure S5 indicate that the RFLR ${ }^{\text {AAAA }}$ mutation does not change the outcome, compared with wild-type protein sequences. Cell death was triggered equally well in Rps $1 \mathrm{~d}$ plants by transient expression of the RFLR ${ }^{\text {AAAA }}$ mutant and wild-type Avr1d protein constructs.

\section{Deletion of the Avrld structural gene causes gain of virulence on Rps $1 \mathrm{~d}$.}

To compare the Avrld genomic region in strains of $P$. sojae that are either virulent or avirulent towards $R p s 1 \mathrm{~d}$, we performed an alignment of sequence reads to the assembled genomic segment (P. sojae V5.0). The sequence reads were from whole-genome shotgun sequencing. The extent of sequence read coverage along the 7-kb segment encompassing the Avrld gene is clearly different between the virulent and avirulent strains of $P$. sojae, as shown in Figure 4. In Rps1d-virulent strains P7074 and P7076, there is no sequence read coverage whatsoever along a $1.53-\mathrm{kb}$ segment that includes the Avrld gene, while this region is covered by sequence reads in the Rps1d-avirulent strains P6497, P7064, and ACR10. This result indicates that gain of virulence to Rps $1 \mathrm{~d}$ and the presence or absence polymorphism of Avrld are due to a $1.53-\mathrm{kb}$ deletion that includes the entire Avrld structural gene.

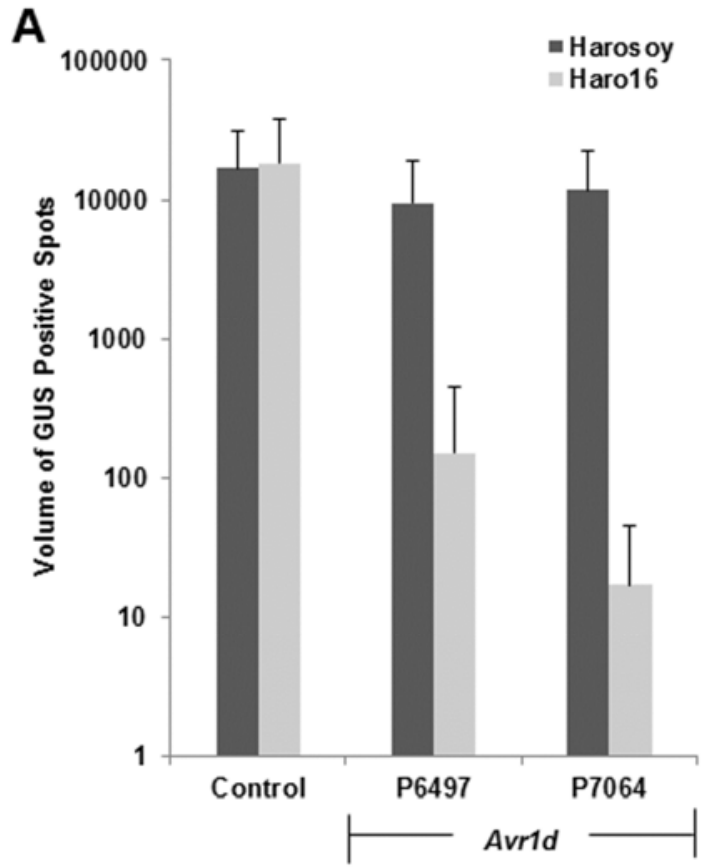

Avr1d binds to phosphatidylinositol 4-phosphate.

Oomycete effector proteins are known to bind to phospholipids, especially PtdIns(3)P. To investigate the affinity of Avr1d towards phospholipids, recombinant protein was produced, purified, and tested by lipid blot and liposome binding assays. Lipid blot assays suggested that Avrld could bind to PtdIns(4)P, so this was further tested and compared with PtdIns(3)P binding by more reliable liposome-based binding methods. Liposomes are made from phosphatidylcholine (PC) and phosphatidylethanolamine (PE), and lesser amounts of additional lipids such as PtdIns(3)P or PtdIns(4)P are added to test specificity. Results shown in Figure 5 indicate that Avr1d has a high affinity for liposomes containing PtdIns(4)P, whereas its binding to liposomes containing PtdIns(3)P did not differ from the binding to control liposomes containing only $\mathrm{PC}$ and PE. A control protein (Vam7pPX) known to bind to PtdIns(3)P displayed affinity for PtdIns(3)P and, to a lesser extent, PtdIns(4)P.

To determine whether the RXLR motif or C-terminal Lys residues are important to the PtdIns(4)P binding of Avr1d, we created two different mutant proteins. In one mutant, the RFLR sequence of Avr1d was mutated to EEEE (Glu-Glu-GluGlu). The other mutant was designed to change three different Lys $(\mathrm{K})$ residues in the C-terminal effector domain to Glu (E), specifically K104E, K105E, and K107E. These residues (K104, K105, and K107) were chosen based on their position in a predicted W-like motif within the effector domain. Results show that the RFLR ${ }^{\text {EEEE }}$ mutations do not affect the phospholipid-binding properties of Avr1d, whereas the K104, K105, and K107E changes drastically altered the lipid-binding characteristics (Fig. 5). Only trace amounts of the K104, K105, and K107E mutant of Avr1d bound to liposomes containing

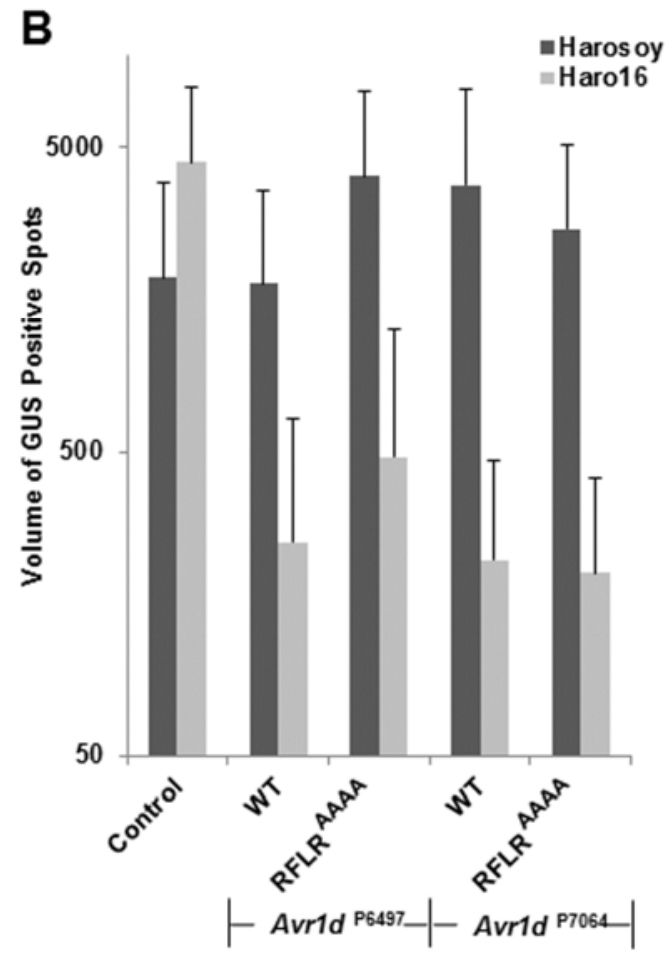

Fig. 3. Measurement of cell death in soybean leaves triggered by transient expression of Avrld. A, Transient expression of Avrld triggers an Rps1d-specific cell death. Leaves of Harosoy ( $r p s 1 \mathrm{~d})$ and Haro16 (Rps 1d) were cobombarded with plasmids carrying a reporter glucoronidase gene (GUS) and a test gene, as indicated. Expression of Avrld alleles from Phytophthora sojae strains P6497 and P7064 is shown (signal peptides were removed from Avrld constructs). Volume intensity of GUS-positive spots was measured from three sections of each leaf, using software. Error bars represent standard deviation for 18 measurements from two independent biological replications. B, Mutation of the Avr1d RXLR motif does not alter the level of cell death for Avrld constructs expressed with native signal peptide. Leaves of Harosoy (rps 1d) and Haro16 (Rps 1d) were cobombarded with plasmids carrying a reporter gene (GUS) and a test gene, as indicated. Expression of the full-length Avr1d with native signal peptide is shown for wild-type (WT) and RXLR-mutated (RFLRAAA) sequences for Avh6 alleles from Phytophthora sojae strains P6497 and P7064. Volume intensity of GUS-positive spots was measured as described above. 
PtdIns(4)P. The K104, K105, and K107E mutant also showed reduced affinity to the PtdIns(3)P liposomes.

\section{DISCUSSION}

Management of soybean root rot caused by $P$. sojae relies on the development of resistant cultivars (Sugimoto et al. 2012). Single dominant Rps genes are powerful resources in breeding programs, but their utility in the field may be defeated by the emergence of new pathogen strains. Molecular characterization of $P$. sojae Avr genes delivers valuable tools to investigate the basis of host resistance and for the development of precise pathogen diagnostic methods (Vleeshouwers et al. 2011). The primary objective of the present study was to identify the $P$. sojae Avrld gene. This was accomplished by genetic mapping of candidate genes selected from a comparative analysis of whole-genome sequence data. Another research group, working independently and using a cross $P$. sojae strain different from that used in our study, has simultaneously provided their

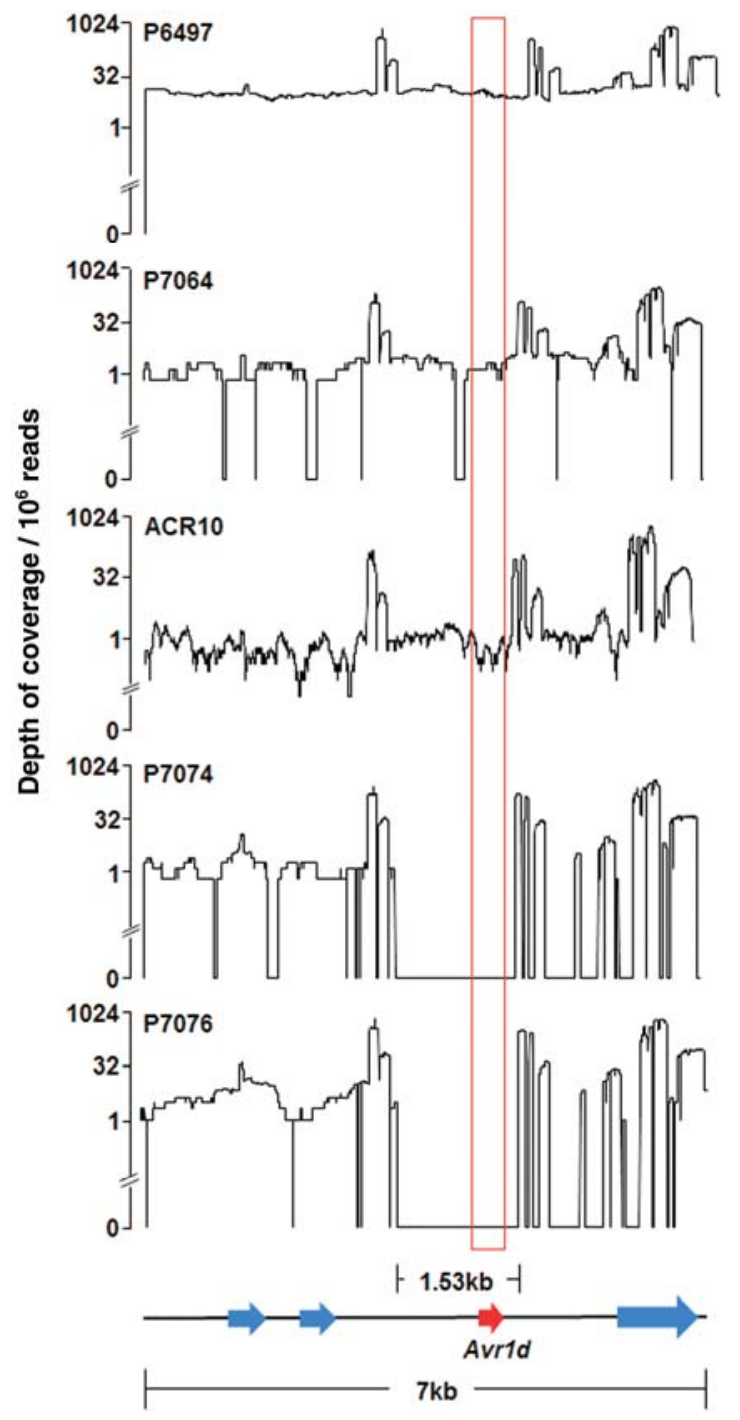

Fig. 4. Sequence read coverage of the Avrld region from five different Phytophthora sojae strains. A 7.0-kb segment containing Avrld and three other predicted genes is from the Phytophthora sojae reference genome assembly V5.0. Raw sequence reads from whole-genome shotgun sequencing of the reference strain (P6497) or from resequencing of strains P7064, ACR10, P7074, and P7076 were aligned to the reference assembly of this segment to determine read coverage. Within the 7.0-kb segment, a $1.53-\mathrm{kb}$ section including the entire Avrld gene is entirely lacking sequence reads in P. soaje strains P7074 and P7076. own experimental evidence that Avh6 corresponds to Avrld (Yin et al. 2013). These separate but matching results provide an added level of confidence in our main conclusion regarding the identity of Avrld.

Previous inheritance studies in $P$. sojae indicated that avirulence to Rps 1d segregates as a single dominant gene (May et al. 2002; Tyler et al. 1995). We confirmed these results in our analysis of progeny from a cross of $P$. sojae strains ACR10 and P7076. Phenotypes conditioned by $P$. sojae Avr genes are inherited as dominant traits, but there are important exceptions. For example, in a cross that was identical to that used in this study, we previously demonstrated non-Mendelian interactions between $A v r 3 a$ alleles resulting from transgenerational effects on gene expression (Qutob et al. 2013). We now show that $P$. sojae ACR10 possesses an Avrld gene that follows Mendelian rules in outcrosses with $\mathrm{P} 7076$. This indicates that the transgenerational effects on Avr3a expression do not affect Avrld and that there is nothing inherently abnormal or dysfunctional in ACR10 $\times$ P70706 progeny with regard to Avr effector gene expression in general.

The selection of candidate $A v h$ genes as an entry point for Avr gene identification in $P$. sojae is now well-established (Dong et al. 2009, 2011a and b). Our choice of 15 candidate
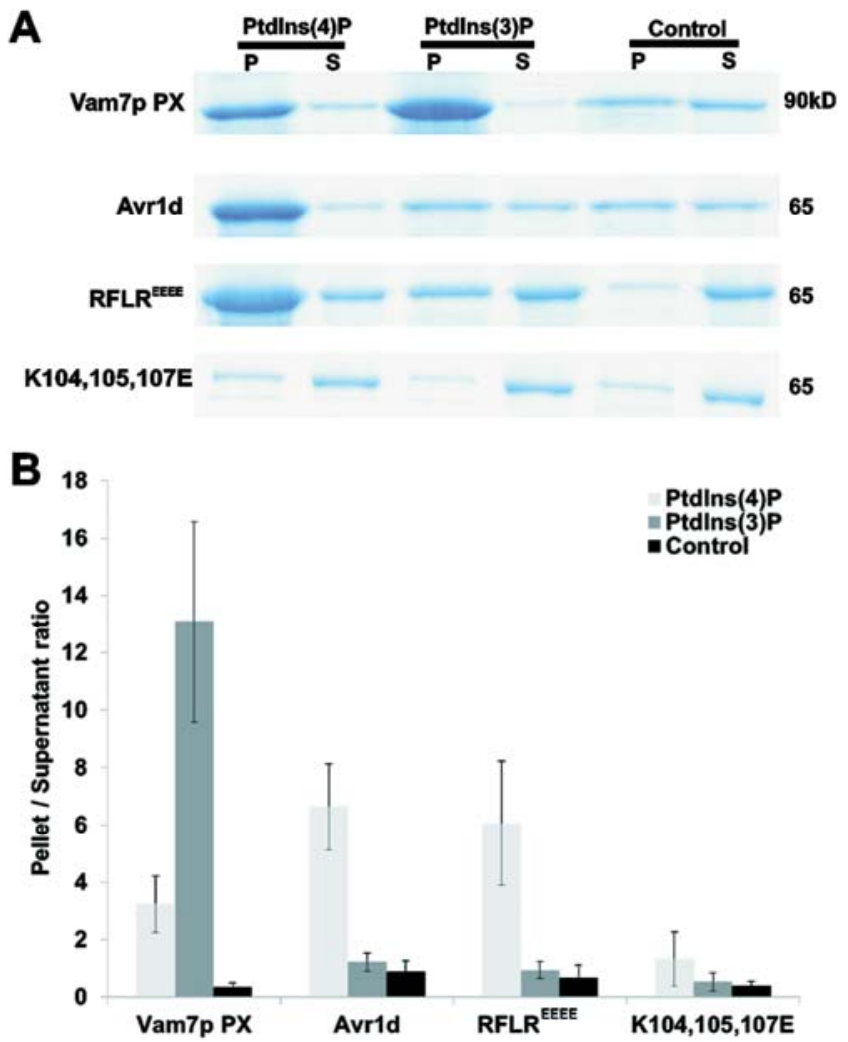

Fig. 5. The effector domain of Avr1d binds to PtdIns(4)P. A, Shown is a sodium dodecyl sulfate-polyacrylamide gel electrophoresis (SDS-PAGE) analysis of a liposome protein-binding assay. Control liposomes or liposomes with added PtdIns(3)P or PtdIns(4)P were prepared and incubated with test proteins as indicated. The Vam7p PX is a control protein known to bind to PtdIns(3)P. Wild-type Avr1d is indicated as Avr1d. Altered versions of Avr1d with mutated RXLR motif or C-terminal Lys residues are indicated as RFLR ${ }^{\mathrm{EEEE}}$ and K104E,105,107E, respectively. After ultracentrifugation the pellet $(\mathrm{P})$ and supernatant $(\mathrm{S})$ fractions were analyzed by SDS-PAGE and proteins were visualized by Coomassie staining. kD indicates the size of proteins as measured in kilodaltons. B, Avr1d binds to PtdIns(4)P. Measurement of liposome-bound versus soluble protein by image analysis of SDS-PAGE results. The ratio of protein in the pellet versus the supernatant fraction is shown. Error bars represent the standard deviation from three independent biological replicates. 
$A v h$ genes was guided by existing sequence and expression data. It was fortunate that Avh6 was among those selected because the candidate gene approach is not an infallible method for discovery of unknown Avr gene targets. Choosing candidate genes relies on imperfect assumptions and incomplete data. Nevertheless, it has the potential to facilitate and accelerate target discovery, as was the case with Avrld.

The presence or absence polymorphism of Avrld among $P$. sojae strains provides another example of the dispensability of most $A v r$ genes and, in particular, of individual RXLR effectors. This illustrates that even highly expressed effectors can be expendable, since Avrld (Avh6) was shown in a previous study to be among the most strongly induced during infection of all the RXLR effectors in the $P$. sojae genome (Wang et al. 2011).

Transient expression analysis by cobombardment with a reporter is a long-standing and widely used technique to evaluate $R$ - and Avr-gene interactions (Allen et al. 2004; Jia et al. 2000; Mindrinos et al. 1994). This method is particularly valuable for analysis of candidate genes in soybean, because its leaves are recalcitrant to infiltration and Agrobacterium-based transformation assays. Expression of each of the two different Avrld alleles in the cobombardment assay triggered cell death but only in plants carrying the Rps $1 \mathrm{~d}$ gene. Thus, the results from this functional test provide a validation of the genetic mapping and haplotype analysis that led to the identification of Avrld. We also performed further cobombardment experiments to test the role of the RXLR motif in the host targeting of Avr1d. Our hypothesis was that mutation of the RXLR motif of Avr1d would reduce cell death triggered by transient expression because host targeting would be impaired in cases in which the signal peptide was retained. Implicit in this assumption is that Rps $1 \mathrm{~d}$ occurs in the cell cytoplasm and that the RXLR motif is important for reentry of secreted protein into the cell. However, the results were negative and the RFLR ${ }^{\mathrm{AAAA}}$ mutation did not reduce cell death to any measureable degree. It is possible that high-level ectopic expression of Avr1d causes translated protein to escape the secretory pathway and directly enter the cytoplasm; or perhaps, Avr1d is efficiently secreted, but reentry of the protein into the cell cytoplasm occurs despite the RFLR $^{\text {AAAA }}$ mutation. Other explanations are possible, especially since the Rps1d gene has not been identified and it is not known where or how ETI is activated by Avr1d. Thus the only conclusion that we can draw from this experiment is that an RFLR $^{\text {AAAA }}$ mutation of Avrld has no effect on its ability to activate ETI under the conditions described.

Mutations of the RXLR motif also failed to alter the lipidbinding properties of Avr1d, as assessed by liposome-binding studies. The binding of recombinant Avr1d to PtdIns(4)P was surprising because other oomycete RXLR effectors have shown a preference for PtdIns(3)P. Each of these phospholipids, PtdIns(4)P and PtdIns(3)P, participate in a variety of cellular functions, including protein trafficking. In animal cells, PtdIns(4)P is enriched in the Golgi apparatus, while PtdIns(3)P is most abundant in the endosomal membrane system (Mayinger 2012). Recent work using murine cells has suggested a role for PtdIns(4)P in maintaining the polyanionic character of membrane lipids that is necessary for electrostatic interactions with basic amino acids of protein cargo (Hammond et al. 2012). The affinity of RXLR effectors towards PtdIns(3)P was initially proposed to be mediated by the RXLR motif itself (Kale et al. 2010), but the consensus now is that this occurs through basic amino acids in the C-terminal domain (Sun et al. 2013; Wawra et al. 2012; Yaeno et al. 2011). There is, however, no such consensus on any hypothesis regarding the significance or biological function of lipid binding by RXLR effectors. Suggestions that lipid binding directs effector targeting in one or both the host or pathogen protein traffick- ing systems are plausible but controversial (Bhattacharjee et al. 2012; Kale et al. 2010; Sun et al. 2013; Wawra et al. 2012; Yaeno et al. 2011). Structural models for several RXLR effectors revealed a common three-helix fold called the WY domain, and this has been proposed as an adaptable scaffold for functional diversification (Win et al. 2012). However, the WY domain represents an unusual example for phosphoinositide binding, and the molecular interactions that coordinate this process are not known (Jiang et al. 2013). Our results show that Avr1d binds to PtdIns(4)P and that this interaction can be disrupted by mutation of Lys residues in the C-terminal domain but not by mutation of the RXLR motif.

In conclusion, we have identified the P. sojae Avrld gene and shown that it encodes an RXLR effector protein. Two alleles of Avrld that differ in predicted amino acid sequence were discovered; however, both alleles activate ETI in soybean plants carrying the Rps1d gene. $P$. sojae strains able to defeat Rps $1 \mathrm{~d}$ lack the Avrld gene entirely. This presence or absence polymorphism of Avrld among $P$. sojae strains is easily measured and will aid diagnostics. These results provide new tools for management of soybean root rot caused by $P$. sojae, a widespread and damaging disease of the world's foremost legume grain crop.

\section{MATERIALS AND METHODS}

\section{Plant materials, Phytophthora sojae strains and virulence scoring.}

Soybean (Glycine max) cultivars Haro16 (Rps1d) and Harosoy (rps1d) from the collection at Agriculture and AgriFood Canada (London, ON, Canada) were used to evaluate the virulence of pathogen cultures. Ten soybean seeds were sown in $10-\mathrm{cm}$ pots (a minimum of three pots per isolate) containing soilless mix (Pro-Mix 'BX', Premier Horticulture Ltd, Rivièredu-Loup, Canada). Pots were watered with $3 \mathrm{mg}$ of 20-20-20 (N-P-K) fertilizer per liter. Plants were grown in a controlled growth chamber with supplementary light (16-h photoperiod with $25^{\circ} \mathrm{C}$ day and $16^{\circ} \mathrm{C}$ night temperatures) for 1 week before inoculations.

Phytophthora sojae isolates were from the collection at Agriculture and Agri-Food Canada. The origin of each strain has been described (Dong et al. 2011a; Qutob et al. 2009). The sexual cross of $P$. sojae strains ACR10 and P7076 and the generation of $F_{1}$ and $F_{2}$ progeny has been described (Qutob et al. 2013). All of the isolates were routinely maintained on $2.5 \%$ (vol/vol) vegetable juice (V8) medium at $25^{\circ} \mathrm{C}$ in the dark. $P$. sojae cultures were transferred to $0.9 \%$ ( $\mathrm{vol} / \mathrm{vol}$ ) V8 agar plates for 5 to 7 days prior to green plant inoculations. Methods of hypocotyl inoculation and virulence scoring have been described (Qutob et al. 2009). Three independent replicates of the virulence assay were performed for each culture tested.

\section{Genetic mapping.}

A total of $40 \mathrm{~F}_{2}$ progeny were selected for genetic mapping of the Avrld interval. Genome sequence data from the reference strain P6497 and from parental strains ACR10 and P7076 were analyzed to design 28 codominant and one dominant DNA marker in the vicinity of the candidate gene Avh6. Details of PCR product sizes in two strains and the restriction enzyme used for each marker are provided in Supplementary Table S1. A selection of 11 of the DNA markers were also scored in 16 different $P$. sojae isolates for the haplotype analysis.

\section{Nucleic acid isolation and RT-PCR.}

The purification of RNA from $P$. sojae mycelium and transcript analysis by RT-PCR was carried out as previously described (Qutob et al. 2000). Total RNA was treated with 
DNAse I (Invitrogen, Carlsbad, CA, U.S.A.). The RT-PCR was conducted on $1 \mu \mathrm{g}$ of RNA, using a reverse transcriptase (SuperScriptIII, Invitrogen) system according to the manufacturer's instructions. Genomic DNA from $P$. sojae mycelium was isolated by phenol chloroform extraction and ethanol precipitation (Tyler et al. 1995). Genomic PCR amplifications were performed using $15 \mathrm{ng}$ of DNA $+0.5 \mu \mathrm{M}$ primers $+0.25 \mathrm{mM}$ dNTPs + Taq + buffer to obtain conditions for PCR and the following program: $94^{\circ} \mathrm{C}$ for $2 \mathrm{~min}, 40$ cycles of $94^{\circ} \mathrm{C}$ for $40 \mathrm{~s}$, $58^{\circ} \mathrm{C}$ for $40 \mathrm{~s}, 72^{\circ} \mathrm{C}$ for 30 to $60 \mathrm{~s}$ (varied with the PCR product size), and a final extension of $72^{\circ} \mathrm{C}$ for $10 \mathrm{~min}$. A $P$. sojae actin gene was used as a control.

\section{Transient expression assays.}

Soybean plants were grown in the growth chambers as described above. Leaves from 14-day-old plants were harvested for cobombardment. The two different Avrld open reading frames (Avh6P6497 and Avh6P7064), but lacking the signal peptide, were amplified using specific primers and cloned into the $35 \mathrm{~S}$ promoter-derived plant expression vector $\mathrm{pFF} 19$, using BamHI and PstI restriction sites. Similarly, constructs for fulllength wild-type Avr1d alleles with signal peptide (FLAvr1dP6497 and FLAvr1dP7064), and the corresponding RFLR-mutated Avr1d synthetic sequences (FLAvr1dP6497RFLR $^{\text {AAAA }}$ and FLAvr1dP7064RFLR ${ }^{\text {AAAA }}$ ) were also cloned into the pFF19 vector, using BamHI and SphI restriction sites, respectively. Soybean leaves of cultivar Harosoy (rps1d) or Haro16 (Rps1d) were transformed by cobombardment with plasmids encoding a GUS reporter gene and a test construct, using tungsten beads. Cobombardment assays were performed as previously described (Qutob et al. 2002, 2006). For the control, the test gene corresponded to a frame-shift mutant of Avrla. Leaves were washed in lactic acid/phenol/water (1:1:1) and were photographed using a digital camera. Sample images were obtained and processed as previously described (Qutob et al. 2009), and the volume of GUS-positive spots was calculated as the intensity (measured in absorbance units) multiplied by the area (square millimeter).

\section{Sequence analysis.}

Genome sequence data for the $P$. sojae reference strain P6497 and resequenced strains P7064, P7074, P7076 were from the Joint Genome Institute database and the VBI Microbial database, respectively. Resequencing of strain ACR10 was performed by The Centre for Applied Genomics (Sick Kids Hospital, Toronto, Canada). Aligning sequence reads from the $P$. sojae strains to a 7-kb segment of reference genome assembly encompassing the Avrld region was performed using the BarrowsWheeler Alignment and Samtools software (Li and Durbin 2009; $\mathrm{Li}$ et al. 2009). Amino acid alignment was determined using software (Lasergene 6.0 MegAlign module). The SignalP 4.0 server was used for secretion signal peptide prediction.

\section{Protein expression and purification.}

For recombinant protein expression, Avr1d from $P$. sojae strain P6497 was defined as wild-type. The RFLR ${ }^{\text {EEEE }}$ and $\mathrm{K} 104$, K105, and K107E mutant sequences were generated and cloned into the pSDK1 vector for expression in Escherichia coli (Kale et al. 2010). The Vacuolar morphogenesis protein 7 Phox homology domain provided a positive control for PdIns(3)P binding. Expression plasmids and protocols were provided by B. Tyler and have been previously described (Kale et al. 2010). Recombinant Avr1d proteins were expressed in fusion with an N-terminal glutathione $S$-transferase and Cterminal green fluorescent protein, and poly-histidine tags. Plasmids were transformed in BL21(DE3) E. coli cells and were grown in Luria-Bertani media at $37^{\circ} \mathrm{C}$, until cells reached an optical density of 0.7 to 0.9 absorbance units. The proteins were induced with $1 \mathrm{mM}$ isopropyl $\beta$-D-1-thiogalactopyranoside, and then, cell growth was continued at $16^{\circ} \mathrm{C}$ for 12 to $16 \mathrm{~h}$. Harvested cells were lysed by sonication in autoclaved chilled lysis buffer (50 mM sodium phosphate, $300 \mathrm{mM}$ sodium chloride, $20 \mathrm{mM}$ imidazole, $\mathrm{pH}$ 8.0). Lysed cells were centrifuged at $10,000 \times g$ for $30 \mathrm{~min}$ at $4^{\circ} \mathrm{C}$. Proteins collected from the supernatant were purified using a column containing $\mathrm{Ni}$ NTA superflow (Qiagen, Basel, Switzerland). The column was then washed $(50 \mathrm{mM}$ sodium phosphate, $300 \mathrm{mM}$ sodium chloride, $60 \mathrm{mM}$ imidazole, $\mathrm{pH} 8.0)$ and was eluted $(50 \mathrm{mM}$ sodium phosphate, $300 \mathrm{mM}$ sodium chloride, $250 \mathrm{mM}$ imidazole, $\mathrm{pH}$ 8.0). Purified proteins were then concentrated by using 10-kDa cut-off centrifugal filter devices (Millipore, Bedford, MA, U.S.A.). Protein concentration was calculated using a microplate reader (Bio-Rad protein assay; Bio-Rad, Munich).

\section{Liposome-protein binding assay.}

Lipids were dissolved in an organic solvent according to the manufacturer's instructions (Cayman Chemical, Ann Arbor, MI, U.S.A.). Liposomes were prepared by mixing equal mass amounts of PC and PE. Additional test lipids PtdIns(3)P or PtdIns(4)P were added to $10 \%$ (wt/wt) concentration. Control liposomes contained only PC and PE. Lipid films were generated by drying the lipid mixture under vacuum overnight at room temperature. Lipid films were hydrated in Tris buffered saline I $(20 \mathrm{mM}$ Tris $\mathrm{HCl}, \mathrm{pH} 6.8,100 \mathrm{mM} \mathrm{NaCl}, 1 \mathrm{mM}$ $\mathrm{NaN}_{3}$ ) to $1 \mathrm{mg} / \mathrm{ml}$ at $68^{\circ} \mathrm{C}$ for $1 \mathrm{~h}$, and then, were subjected to three freeze-thaw cycles (immersion in liquid nitrogen, then thawed at $25^{\circ} \mathrm{C}$ ). Large unilamellar vesicles were formed by extruding the lipid suspension through an Avanti extruder with a $0.2-\mu \mathrm{m}$ filter at $68^{\circ} \mathrm{C}$ (20 cycles). Liposomes were collected by centrifugation at $40,000 \times g$ for 20 min using an ultracentrifuge and were resuspended in Tris-buffered saline II $(20 \mathrm{mM}$ Tris $\mathrm{HCl}, \mathrm{pH} 5.8,50 \mathrm{mM} \mathrm{NaCl}, 1 \mathrm{mM} \mathrm{NaN}_{3}$ ) to a final concentration of $5 \mu \mathrm{g} / \mu \mathrm{l}$. Proteins were centrifuged at $40,000 \times g$ for $20 \mathrm{~min}$ at $4^{\circ} \mathrm{C}$ to remove protein aggregates. Purified protein $(10 \mu \mathrm{g})$ was incubated with $200 \mu \mathrm{g}$ of liposome for 30 $\min$ at $4^{\circ} \mathrm{C}$. Protein-liposome mixtures were centrifuged at $40,000 \times g$ for $20 \mathrm{~min}$ at $4^{\circ} \mathrm{C}$. Pellets containing liposomebound proteins and supernatants containing free proteins were then analyzed by sodium dodecyl sulfate polyacrylamide gel electrophoresis and were stained with Coomassie-blue using standard methods.

\section{ACKNOWLEDGMENTS}

We thank B. P. Chapman, A. Gaidauskas-Scott, and K. Kuflu for contributions to this work; B. Tyler for sharing information and materials and for hosting R. Na in a training workshop for lipid binding assays; and L. Tian for use of the particle bombardment apparatus. Research funded by Agriculture and Agri-Food Canada GRDI; R. Na and D. Yu supported in part by scholarships from the China Ministry of Education.

\section{LITERATURE CITED}

Allen, R. L., Bittner-Eddy, P. D., Grenville-Briggs, L. J., Meitz, J. C., Rehmany, A. P., Rose, L. E., and Beynon, J. L. 2004. Host-parasite coevolutionary conflict between Arabidopsis and downy mildew. Science 306:1957-1960.

Bhattacharjee, S., Stahelin, R. V., Speicher, K. D., Speicher, D. W., and Haldar, K. 2012. Endoplasmic reticulum PI(3)P lipid binding targets malaria proteins to the host cell. Cell 148:201-212.

Bozkurt, T. O., Schornack, S., Banfield, M. J., and Kamoun, S. 2012. Oomycetes, effectors, and all that jazz. Curr. Opin. Plant Biol. 15:483-492

Dong, S., Qutob, D., Tedman-Jones, J., Kuflu, K., Wang, Y., Tyler, B. M., and Gijzen, M. 2009. The Phytophthora sojae avirulence locus Avr3c encodes a multi-copy RXLR effector with sequence polymorphisms among pathogen strains. PLoS One 4:e5556. Published online.

Dong, S., Yu, D., Cui, L., Qutob, D., Tedman-Jones, J., Kale, S. D., 
Tyler, B. M., Wang, Y., and Gijzen, M. 2011a. Sequence variants of the Phytophthora sojae RXLR effector Avr3a/5 are differentially recognized by Rps3a and Rps5 in soybean. PLoS One 6:e20172. Published online.

Dong, S., Yin, W., Kong, G., Yang, X., Qutob, D., Chen, Q., Kale, S. D., Sui, Y., Zhang, Z., and Dou, D. 2011b. Phytophthora sojae avirulence effector $A v r 3 b$ is a secreted NADH and ADP-ribose pyrophosphorylase that modulates plant immunity. PLoS Path 7:e1002353. Published online.

Dou, D., Kale, S. D., Wang, X., Jiang, R. H. Y., Bruce, N. A., Arredondo, F. D., Zhang, X., and Tyler, B. M. 2008. RXLR-mediated entry of Phytophthora sojae effector Avr1b into soybean cells does not require pathogen-encoded machinery. Plant Cell 20:1930-1947.

Dou, D., Kale, S. D., Liu, T., Tang, Q., Wang, X., Arredondo, F. D., Basnayake, S., Whisson, S., Drenth, A., Maclean, D., and Tyler, B. M. 2010. Different domains of Phytophthora sojae effector Avr $4 / 6$ are recognized by soybean resistance genes Rps4 and Rps6. Mol. PlantMicrobe Interact. 23:425-435.

Gijzen, M., and Qutob, D. 2009. Phytophthora sojae and soybean. Pages 303-331 in Oomycete Genetics and Genomics: Diversity, Interactions, and Research Tools, K. Lamour and S. Kamoun, eds. Wiley-Blackwell, Hoboken, NJ, U.S.A

Haas, B. J., Kamoun, S., Zody, M. C., Jiang, R. H. Y., Handsaker, R. E., Cano, L. M., Grabherr, M., Kodira, C. D., Raffaele, S., and Torto-Alalibo, T. 2009. Genome sequence and analysis of the Irish potato famine pathogen Phytophthora infestans. Nature 461:393-398.

Hammond, G. R. V., Fischer, M. J., Anderson, K. E., Holdich, J., Koteci, A., Balla, T., and Irvine, R. F. 2012. PI4P and PI(4,5)P2 are essential but independent lipid determinants of membrane identity. Science Signalling 337:727-730

Jia, Y., McAdams, S. A., Bryan, G. T., Hershey, H. P., and Valent, B. 2000. Direct interaction of resistance gene and avirulence gene products confers rice blast resistance. EMBO (Eur. Mol. Biol. Organ.) J. 19:40044014.

Jiang, R. H., Stahelin, R. V., Bhattacharjee, S., and Haldar, K., 2013. Eukaryotic virulence determinants utilize phosphoinositides at the ER and host cell surface. Trends Microbiol. 21:145-156.

Jiang, R. H. Y., Tripathy, S., Govers, F., and Tyler, B. M. 2008. RXLR effector reservoir in two Phytophthora species is dominated by a single rapidly evolving superfamily with more than 700 members. Proc. Natl. Acad. Sci. U.S.A. 105:4874.

Jones, J. D. G., and Dangl, J. L. 2006. The plant immune system. Nature 444:323-329.

Kale, S. D., Gu, B., Capelluto, D. G. S., Dou, D., Feldman, E., Rumore, A., Arredondo, F. D., Hanlon, R., Fudal, I., and Rouxel, T. 2010. External lipid PI3P mediates entry of eukaryotic pathogen effectors into plant and animal host cells. Cell 142:284-295.

Li, H., and Durbin, R. 2009. Fast and accurate short read alignment with Burrows-Wheeler transform. Bioinformatics 25:1754-1760.

Li, H., Handsaker, B., Wysoker, A., Fennell, T., Ruan, J., Homer, N., Marth, G., Abecasis, G., and Durbin, R. 2009. The sequence alignment/map format and SAMtools. Bioinformatics 25:2078-2079.

May, K., Whisson, S., Zwart, R., Searle, I., Irwin, J., Maclean, D., Carroll, B., and Drenth, A. 2002. Inheritance and mapping of 11 avirulence genes in Phytophthora sojae. Fungal Gen. Biol. 37:1-12.

Mayinger, P. 2012. Phosphoinositides and vesicular membrane traffic Biochem. Biophys. Acta 1821:1104-1113.

Mindrinos, M., Katagiri, F., Yu, G.L., and Ausubel, F. M. 1994. The A. thaliana disease resistance gene RPS2 encodes a protein containing a nucleotide-binding site and leucine-rich repeats. Cell 78:1089-1099.

Qutob, D., Hraber, P. T., Sobral, B. W. S., and Gijzen, M. 2000. Comparative analysis of expressed sequences in Phytophthora sojae. Plant Physiol. 123:243-254.

Qutob, D., Kamoun, S., and Gijzen, M. 2002. Expression of a Phytophthora sojae necrosis-inducing protein occurs during transition from biotrophy to necrotrophy. Plant J. 32:361-373.

Qutob, D., Kemmerling, B., Brunner, F., Küfner, I., Engelhardt, S., Gust, A. A., Luberacki, B., Seitz, H. U., Stahl, D., and Rauhut, T. 2006. Phytotoxicity and innate immune responses induced by Nep1-like proteins. Plant Cell 18:3721-3744

Qutob, D., Tedman-Jones, J., Dong, S., Kuflu, K., Pham, H., Wang, Y., Dou, D., Kale, S. D., Arredondo, F. D., and Tyler, B. M. 2009. Copy number variation and transcriptional polymorphisms of Phytophthora sojae RXLR effector genes Avrla and Avr3a. PLoS One 4:e5066. Published online.
Qutob, D., Chapman, B. P., and Gijzen, M. 2013. Transgenerational gene silencing causes gain of virulence in a plant pathogen. Nature Comm. 4:1439.

Rietman, H., Bijsterbosch, G., Cano, L. M., Lee, H.-R., Vossen, J. H., Jacobsen, E., Visser, R. G. F., Kamoun, S., and Vleeshouwers, V. G. A. A. 2012. Qualitative and quantitative late blight resistance in the potato cultivar Sarpo Mira is determined by the perception of five distinct RXLR effectors. Mol. Plant-Microbe Interact. 25:910-919.

Shan, W., Cao, M., Leung, D., and Tyler, B. M. 2004. The Avrlb locus of Phytophthora sojae encodes an elicitor and a regulator required for avirulence on soybean plants carrying resistance gene Rps $1 b$. Mol. Plant-Microbe Interact. 17:394-403.

Sugimoto, T., Kato, M., Yoshida, S., Matsumoto, I., Kobayashi, T., Kaga, A., Hajika, M., Yamamoto, R., Watanabe, K., and Aino, M. 2012. Pathogenic diversity of Phytophthora sojae and breeding strategies to develop Phytophthora-resistant soybeans. Breeding Sci. 61:511522.

Sun, F., Kale, S. D., Azurmendi, H. F., Li, D., Tyler B. M., and Capelluto, D. G. 2013. Structural basis for interactions of the Phytophthora sojae RxLR effector Avh5 with phosphatidylinositol 3-phosphate and for host cell entry. Mol. Plant-Microbe Interact. 26:330-344.

Thomma, B. P. H. J., Nürnberger, T., and Joosten, M. H. A. J. 2011. Of PAMPs and effectors: The blurred PTI-ETI dichotomy. Plant Cell 23:415

Tyler, B. M., Forster, H., and Coffey, M. D. 1995. Inheritance of avirulence factors and restriction fragment length polymorphism markers in outcrosses of the oomycete Phytophthora sojae. Mol. Plant-Microbe Interact. 8:515-523.

Tyler, B. M., Tripathy, S., Zhang, X., Dehal, P., Jiang, R. H. Y., Aerts, A., Arredondo, F. D., Baxter, L., Bensasson, D., and Beynon, J. L. 2006 Phytophthora genome sequences uncover evolutionary origins and mechanisms of pathogenesis. Science 313:1261-1266.

Vleeshouwers, V. G. A. A., Raffaele, S., Vossen, J. H., Champouret, N. Oliva, R., Segretin, M. E., Rietman, H., Cano, L. M., Lokossou, A., and Kessel, G. 2011. Understanding and exploiting late blight resistance in the age of effectors. Ann. Rev. Phytopath. 49:507-531.

Wang, Q., Han, C., Ferreira, A. O., Yu, X., Ye, W., Tripathy, S., Kale, S. D., Gu, B., Sheng, Y., and Sui, Y. 2011. Transcriptional programming and functional interactions within the Phytophthora sojae RXLR effector repertoire. Plant Cell 23:2064-2086.

Wawra, S., Agacan, M., Boddey, J. A., Davidson, I., Gachon, C. M., Zanda, M., Grouffaud, S., Whisson, S. C., Birch, P. R. J., Porter, A. J. and van West P. 2012. Avirulence protein 3a (AVR3a) from the potato pathogen Phytophthora infestans, forms homodimers through its predicted translocation region and does not specifically bind phospholipids. J. Biol. Chem. 287:38101-38109.

Whisson, S. C., Boevink, P. C., Moleleki, L., Avrova, A. O., Morales, J. G., Gilroy, E. M., Armstrong, M. R., Grouffaud, S., van West, P., and Chapman, S. 2007. A translocation signal for delivery of oomycete effector proteins into host plant cells. Nature 450:115-118.

Win, J., Morgan, W., Bos, J., Krasileva, K. V., Cano, L. M., ChaparroGarcia, A., Ammar, R., Staskawicz, B. J., and Kamoun, S. 2007. Adaptive evolution has targeted the C-terminal domain of the RXLR effectors of plant pathogenic oomycetes. Plant Cell 19:2349-2369.

Win, J., Krasileva, K. V., Kamoun, S., Shirasu, K., Staskawicz, B. J., and Banfield, M. J., 2012. Sequence divergent RXLR effectors share a structural fold conserved across plant pathogenic oomycete species. PLoS Pathog. 8:e1002400. Published online.

Yaeno, T., Li, H., Chaparro-Garcia, A., Schornack, S., Koshiba, S. Watanabe, S., Kigawa, T., Kamoun, S., and Shirasu, K. 2011. Phosphatidylinositol monophosphate-binding interface in the oomycete RXLR effector AVR3a is required for its stability in host cells to modulate plant immunity. Proc. Natl. Acad. Sci. U.S.A. 108:14682-14687.

Yin, W., Dong, S., Zhai1, L., Lin, Y., Zheng, X., and Wang, Y. 2013. Phytophthora sojae Avrld genes encode an RxLR-dEER effector with presence and absence polymorphisms among pathogen strains. Mol Plant-Microbe Interact. 26: 958-968.

\section{AUTHOR-RECOMMENDED INTERNET RESOURCES}

Joint Genome Institute database: genome.jgi-psf.org SignalP 4.0 server: www.cbs.dtu.dk/services/SignalP Virginia Bioinformatics Institute microbial database: www.vbi.vt.edu/resources_and tools 